Conclusion. Psychiatric Frequent Attenders have complex needs, which do not fit neatly into existing psychiatric diagnoses and services.

The high frequency of emotional trauma, substance misuse and personality disorder indicates a need for training of clinicians in these services to manage these patients, as well as planning for referral pathways for this group of patients who provide services with major challenges in appropriate pathways to care and follow-up

How do people with dementia present to the services, and why do they present late? A descriptive study in a Tertiary Care Hospital in Sri Lanka

Malsha Gunathilake ${ }^{1 \star}$ and Chathurie Suraweera ${ }^{2}$

${ }^{1}$ North East London Foundation Trust and ${ }^{2}$ Faculty of Medicine,

University of Colombo

${ }^{\star}$ Corresponding author.

doi: 10.1192/bjo.2021.677

Aims. To assess how patients with dementia present to services and reasons for delayed presentation among patients with dementia in Sri Lanka.

Method. A descriptive cross-sectional study was conducted among 83 newly diagnosed patients with dementia and their caregivers at the University Psychiatry Unit, National Hospital of Sri Lanka. They were interviewed using a semi-structured pre-tested questionnaire. Statistical Package for the Social Sciences (SPSS) was utilized for data analysis.

Result. The mean age of the patients was $71.53(\mathrm{SD}=7.595)$ years. The commonest type of dementia in the cohort was Alzheimer's disease $(\mathrm{N}=49,59 \%)$. The mean untreated duration before the first presentation was $16.33(\mathrm{SD}=16.13)$ months. A family member or the care-giver had initiated help-seeking in many $(\mathrm{N}=65,78.3 \%)$. $84.33 \%$ of patients had behavioural and Psychological Symptoms of Dementia (BPSD) at first presentation. BPSD was the main reason for help-seeking in 40(48.2\%) cases. Among them, psychosis $(\mathrm{n}=18,45 \%)$, depression $(\mathrm{n}=9,22.5 \%)$, disinhibition $(\mathrm{n}=4,10 \%)$ and wandering $(\mathrm{n}=3,7.5 \%)$ were common.

Lack of awareness on dementia $(n=70,93.3 \%$ and $n=68,86.1 \%)$ and considering cognitive impairment as a normal part of ageing ( $\mathrm{n}$ $=39,52 \%$ and $\mathrm{n}=43,54.4 \%$ )were the commonest reasons for delayed presentation reported by patients and care-givers respectively. Twelve patients misattributed the symptoms to their existing medical or psychiatric conditions. The mean untreated duration was significantly higher in the patient group with a family history of dementia (30.5 months) compared to those without a family history (12.8 months)(t $=3.818 ; \mathrm{p}=0.000)$. Similarly, the mean untreated duration was significantly higher when there is a family history of dementia among the caregivers (25.53months) compared to the group of care-givers without a family history $(13.85$ months $)(t=2.532 ; \mathrm{p}=0.013)$. Age, sex, education, occupation, income, knowledge on dementia of the patients and the caregivers, illness-related characteristics (type, severity, and presence of BPSD) or being in contact with medical services were not significantly associated with the timing of the first presentation.

Conclusion. There is a delay of more than one year for patients with dementia to present to services in Sri Lanka. The commonest reason for the presentation is BPSD. Lack of prior awareness of dementia and considering the cognitive impairment as a part of normal ageing by both patients and carers were the main reasons for delayed presentation. Patients with a family history of dementia present late than those without a family history. There is no significant association between the timing of presentation and the socio-demographic factors of the patients and care-givers, the presence of prior knowledge on dementia, illness-related characteristics, or contact with medical services.
Evaluating participant experience in Balint online sessions held during the COVID-19 pandemic lessons learnt and moving forward

\author{
Nikhita Handa ${ }^{1 *}$, Romy Garbutt ${ }^{1}$ and Sylvia Chudley ${ }^{2}$ \\ ${ }^{1}$ East Lancashire Hospitals NHS Trust and ${ }^{2}$ The Balint Society \\ ${ }^{\star}$ Corresponding author.
}

doi: 10.1192/bjo.2021.678

Aims. From the outset of the COVID-19 global pandemic and the lockdown that subsequently ensued, a challenge was posed to reshape previously face-to-face meetings in all walks of life. One area that rose to this, with quick introduction of online sessions, was the Balint Group. We aimed to take a snapshot of the effect virtual Balint sessions have had and analyse the themes that members of virtual Balint groups have been identifying about their online group experience at this particularly challenging time for healthcare workers. We hope this will inform both leaders and participants of future online groups of the benefits and pitfalls found by these members reflecting on their first experiences of virtual Balint.

Method. Seven members of virtual Balint groups across the UK were randomly selected for interview from a pool of volunteers facilitated by the UK Balint Society after the first 6 months of their first virtual Balint experience. Interviews were conducted by two academic foundation doctors who were not members of the Balint groups. Qualitative thematic analysis was then conducted on these interview transcripts. Going forward, as Balint groups continue online, the researchers plan to interview further group members and leaders to look for change and development in the primary themes identified. Result. Key positive themes identified when discussing virtual Balint were ease of access, increased anonymity, attention to facial expressions and interaction with participants from different parts of the country. The most common drawback themes were a lack of socialising and different group dynamic as well as the expected technical and environmental challenges. Interestingly all participants reported that 'silence' and 'sitting/stepping back' were still used in their online sessions. Core theme analysis indicates the virtual Balint descriptions draw out sentiments of safe, open and structured sessions. In these early sessions a frequent theme was the increased role of the leader.

Conclusion. All participants interviewed so far have felt their online experiences have had many positive aspects. They highlight areas they feel virtual Balint could develop to better replicate the original sessions. The fact some interviewees would prefer to maintain online Balint groups even when 'in person' options resume makes it likely this will not be a transient rise in virtual Balint and that the style may be here to stay. Based on this, the role for feedback and constant evaluation and improvement will be central to virtual Balint evolution.

The impact of the COVID-19 pandemic on symptom subtypes of obsessive-compulsive disorder: a cross-sectional study

Athanasios Hassoulas ${ }^{1 \star}$, Katja Umla-Runge ${ }^{1}$, Olivia Adams ${ }^{1}$, Madeline Scurlock-Green ${ }^{1}$, Abeer Zahid ${ }^{1}$, Antonia Hassoulas ${ }^{1}$ and Eliana Panayiotou ${ }^{2}$

${ }^{1}$ Cardiff University School of Medicine and ${ }^{2}$ Swansea Bay University Health Board

${ }^{*}$ Corresponding author.

doi: 10.1192/bjo.2021.679

Aims. Since the COVID-19 outbreak was declared a global pandemic, public health messages have emphasised the importance 
of frequent handwashing in limiting the transmission of the virus. Whilst crucial in controlling transmission, such messaging may have an adverse effect on individuals with OCD. The primary aim of this study was to investigate any significant changes to handwashing behaviour, as well as other related hygiene behaviours, across all symptom dimensions of OCD. The frequency of engaging with pandemic-related media coverage was also considered across all symptom subtypes.

Method. A cross-sectional study was conducted, with a total of 332 participants recruited. Participants who scored above the optimal cut-off score on the Obsessive-Compulsive Inventory Revised edition (OCI-R) were included in the analysis $(\mathrm{n}=254)$. Scores on the six subscales of the OCI-R were correlated with responses to a COVID-19 Impact measure.

Result. Factor analysis of the COVID-19 Impact measure revealed that items loaded on two components of the measure (handwashing and distress-avoidance). A significant correlation was revealed between the handwashing component and the OCI-R washing subscale ( $\mathrm{rs}=0.523, \mathrm{p}=0.0001$ ), as well as between distressavoidance and the OCI-R washing and ordering subscales $(\mathrm{s}=-0.227, \mathrm{p}=0.0001 ; \mathrm{rs}=-0.159, \mathrm{p}=0.006)$. Content analysis revealed disruption to treatment delivery and worsening symptom severity in participants with contamination-related OCD.

Conclusion. The pandemic has had a significant impact on individuals with contamination-related OCD symptoms, in relation to symptom severity and treatment disruption. Consideration should be given to targeted support tailored to patients with this subtype of OCD.

Person centred approaches to learning hold a potential for a mature depth of understanding and engagement as opposed to the traditional 'transmission of knowledge' approach to learning

Catherine Hayes $^{1 \star}$ and Adrian Heald ${ }^{2}$

${ }^{1}$ University of Nottingham and ${ }^{2}$ University of Manchester, Salford Royal Hospital

${ }^{\star}$ Corresponding author.

doi: 10.1192/bjo.2021.680

Aims. Do students experience a person-centred experiential (PCE) approach to learning in a university context differently to transmitted knowledge learning from prior education, and if so, how?

Background. The person-centred approach, as defined and developed by Carl Ransom Rogers, remains on the margins of practice in the UK. The approach sustains a non-medical stance. All of the Improving Access to Psychological Therapies Person Centred Experiential Counselling (APT PCEC) workforce require a qualification in person-centred experiential counselling. In order to attune to Roger's hypothesis regarding the conditions required in order to facilitate psychological growth, person-centred learning is a principle stance.

Researching experiences of PCE learning through anonymous feedback from students attending different levels of training (BA, MA and post qualification PCE-Counselling for Depress (CfD) License) is an initial test of the hypothesis .

Counselling education in the UK is increasingly highly standardised and driven by competency frameworks. This work begins to uncover person-centred students' evaluation of undertaking person-centred qualifications. Modules and continuing professional practice were constructed to facilitate a person-centred learning environment wherein the curriculum was designed by students or the experiential aspect of the learning drove the agenda
Method. The sample was made up of $(\mathrm{N}=30)$ students. 8 students were studying for a Master's degree in person-centred experiential psychotherapy, 10 students were studying a BA in humanistic psychotherapy, 12 students were attending a mandatory IAPT Continuous Professional Development (CPD) training in PCE therapy. The evaluation responses were subject to a thematic analysis.

Result. The emerging themes parallel each other and indicate that degree students were very aware of the difference from their previous learning experience in education.

$68 \%$ of MA Students experienced psychological maturation through the process of training.

$83 \%$ of BA students became more agentic in their approach to learning.

83\% IAPT therapists noticed the nurturing, compassion and humane approach to the learning, despite the mandatory nature of the offer and empowered them in regards to their non-medical stance within an NHS context.

Conclusion. Our findings point to the significance and impact of person-centred learning for person-centred psychotherapists' development during and post-qualification. Implications can be drawn in regards to engaging with person-centred learning in public sector and health contexts.

Person centred approaches to learning hold a potential for a mature depth of understanding and engagement as opposed to the traditional 'transmission of knowledge' approach to learning.

\section{Reducing fatigue-related symptoms in Long COVID-19: finding an intervention that works}

Adrian Heald ${ }^{1 \star}$, Lisa Riste ${ }^{2}$, Andreas Walther ${ }^{3}$, Mike Stedman $^{4}$, Annice Mukherjee ${ }^{2}$ and Ray Perrin ${ }^{2}$

${ }^{1}$ Salford Royal Foundation Trust; ${ }^{2}$ University of Manchester;

${ }^{3}$ University of Zurich and ${ }^{4}$ Res Consortium

${ }^{*}$ Corresponding author.

doi: 10.1192/bjo.2021.681

Aims. In the early days of the first global wave of the COVID-19 pandemic, the potential for a post-viral syndrome to manifest following COVID-19 infection was highlighted.

It was pointed out that an early intervention applying management techniques used in patients with CFS/ME appeared to help reduce the fatigue related symptoms of Long COVID.

Here we present an analysis of a consecutive case series of the first twenty patients' data collected. Our aim was to evaluate the potential of this mode of treatment for Long COVID.

Method. Face to face treatment sessions with the practitioners occurred once a week, involving effleurage and other manual articulatory techniques.

The individuals being treated also undertook a daily selfmassage along with gentle mobility exercises and alternating warm and cool gel packs on the upper spine, to encourage a reduction of spinal inflammation and further aid lymph drainage of the brain and spine.

Symptom severity was recorded using the self-reported 54-item Profile of Fatigue Related States (PFRS).

Result. The mean age of the men was 41.8 years with a range of 29.1-53.1 years with the corresponding mean age for women being 39.3 years with a range of 28.3-50.4 years.

The average time interval between onset of Coronavirus symptoms and start of treatment for Long COVID was just over 20 weeks. The average number of treatment sessions was similar at 9.7 in men and 9.4 in women. 